\title{
Integration of bacterial expansin-like proteins into cellulosome promotes the cellulose degradation
}

\author{
Chao Chen ${ }^{1,2,3,4} \cdot$ Zhenling Cui $^{1,2,3,4}$ - Xiangfei Song ${ }^{2,3}$. \\ Ya-Jun Liu ${ }^{1,2,3}$ - Qiu Cui ${ }^{1,2,3}$ • Yingang Feng ${ }^{1,2,3}$ (1)
}

Received: 10 July 2015 / Revised: 2 October 2015 / Accepted: 7 October 2015 / Published online: 31 October 2015

(C) Springer-Verlag Berlin Heidelberg 2015

\begin{abstract}
Cellulosomes are multi-enzyme complexes assembled by cellulases and hemicellulases through dockerin-cohesin interactions, which are the most efficient system for the degradation of lignocellulosic resources in nature. Recent genomic analysis of a cellulosome-producing anaerobe Clostridium clariflavum DSM 19732 revealed that two expansin-like proteins, Clocl_1298 and Clocl_1862, contain a dockerin module, which suggests that they are components of the cellulosome. Bacterial expansin-like proteins do not have hydrolytic activities, but can facilitate the degradation of cellulosic biomass via synergistic effects with cellulases. In this study, the synergistic effect of the expansin-like proteins with both native and designer cellulosomes was investigated. The free expansin-like proteins, including expansin-like domains of Clocl_1298 and Clocl_1862,
\end{abstract}

Electronic supplementary material The online version of this article (doi:10.1007/s00253-015-7071-6) contains supplementary material, which is available to authorized users.

Qiu Cui

cuiqiu@qibebt.ac.cn

Yingang Feng

fengyg@qibebt.ac.cn

1 Qingdao Engineering Laboratory of Single Cell Oil, Qingdao Institute of Bioenergy and Bioprocess Technology, Chinese Academy of Sciences, 189 Songling Road, Qingdao, Shandong 266101, China

2 Shandong Provincial Key Laboratory of Energy Genetics, Qingdao Institute of Bioenergy and Bioprocess Technology, Chinese Academy of Sciences, 189 Songling Road, Qingdao, Shandong 266101, China

3 CAS Key Laboratory of Biofuels, Qingdao Institute of Bioenergy and Bioprocess Technology, Chinese Academy of Sciences, 189 Songling Road, Qingdao, Shandong 266101, China

4 University of Chinese Academy of Sciences, Beijing 100049, China as well as a well-studied bacterial expansin-like protein BsEXLX1 from Bacillus subtilis, promoted the cellulose degradation by native cellulosomes, indicating the cellulosomal expansin-like proteins have the synergistic function. When they were integrated into a trivalent designer cellulosome, the synergistic effect was further amplified. The sequence and structure analyses indicated that these cellulosomal expansin-like proteins share the conserved functional mechanism with other bacterial expansin-like proteins. These results indicated that non-catalytic expansin-like proteins in the cellulosome can enhance the activity of the cellulosome in lignocellulose degradation. The involvement of functional expansin-like proteins in the cellulosome also implies new physiological functions of bacterial expansin-like proteins and cellulosomes.

Keywords Expansin - Cellulosome · Cellulose hydrolysis · Dockerin $\cdot$ Clostridium clariflavum

\section{Introduction}

The biomass of plant cell walls is a great potential resource for the production of biofuel because of its abundant reserve and inexpensive price in the biosphere. However, the plant cell walls are well protected from chemical and biochemical attacks by a hemicellulose and lignin complex (Kumar et al. 2008), which decreases its hydrolysis rate and limits its conversion into fuels. Many anaerobic cellulolytic organisms such as the species of Clostridium can degrade the plant cell wall substrates efficiently using a multi-enzyme complex termed cellulosome, which generally contains a scaffoldin and multiple enzymes. The scaffoldin consists of several tandem repeated cohesin modules, while cellulosomal enzymes always contain a dockerin module which can bind to one of the cohesin modules in the scaffoldin through the specific cohesin-dockerin interaction (Bayer et al. 2004, 
2008). The assembly of enzymes into the cellulosome would enhance their synergistic and conjugative interactions (Fierobe et al. 2002; Demain et al. 2005). Thus, the cellulosome is considered one of the most effective strategies for the bioconversion of lignocellulose. In addition to the cellulolytic enzymes and scaffoldins, the cellulosome also contains other dockerinbearing components (Zverlov et al. 2005; Raman et al. 2009), such as protease and protease inhibitor which has been extensively studied (Meguro et al. 2011; Cuiv et al. 2013; Xu et al. 2014). However, other function-unknown proteins with dockerin modules are less studied.

Clostridium clariflavum DSM 19732 is a typical cellulolytic thermophilic anaerobe. According to the genome sequencing results (Izquierdo et al. 2012), C. clariflavum DSM 19732 contains cellulosomal genes similar, but more complicated, to the genes of Clostridium thermocellum, the most widely studied cellulosome-producing bacterium. Two genes encoding expansin-like proteins were found in $C$. clariflavum DSM 19732 by genome mining, and both of them contain a dockerin module, indicating that they are components of the cellulosome (Artzi et al. 2014). Expansins are plant cell wall proteins involved in the wall loosening, playing key roles in the plant cell growth and other developmental processes, such as pollination, fruit ripening, organ abscission, and seed germination (Cosgrove 2000). Many expansin-like proteins have been discovered in bacteria (Lee et al. 2013; Bunterngsook et al. 2014, 2015; Georgelis et al. 2015). BsEXLX1 from Bacillus subtilis is the first bacterial expansin-like protein recognized by virtue of its structural and functional similarity to plant expansins (Kerff et al. 2008; Kim et al. 2008). Most of bacterial expansin-like proteins facilitate the microbial colonization of plants, with consequences for pathogen virulence and biocontrol (Kim et al. 2013; Georgelis et al. 2014; Nikolaidis et al. 2014; Suzuki et al. 2014). Expansin and expansin-like proteins were found to disrupt hydrogen bonding between cellulose microfibrils and other cell wall polysaccharides without hydrolytic activity, and their disruption activity confers a synergistic effect to the enzymatic hydrolysis of cellulose and xylan by enabling it to be more accessible to the enzyme (Kim et al. 2009; Lee et al. 2013), although some contradicted results were reported by several studies which argued that the synergistic effects of bacterial expansins are non-specific effects (Georgelis et al. 2015). Therefore, bacterial expansin-like proteins attract researchers and engineers by their synergistic effect in the conversion of cellulosic biomass to sugars. Although bacterial expansin-like proteins have been widely discovered and extensively studied, those in C. clariflavum are firstly discovered as cellulosomal expansin-like proteins. It is interesting whether the cellulosomal expansin-like proteins could provide further synergistic effect compared to free expansin-like proteins as a new method to enhance the hydrolysis efficiency of cellulosome.
In this study, we first investigated the synergistic effect of the two expansin-like proteins Clocl_1298 and Clocl_1862 with the native cellulosomes from C. clariflavum DSM 19732 and $C$. thermocellum ATCC 27405, to determine whether the two proteins indeed possessed the capacity to promote the cellulose degradation similar to $B s E X L X 1$. We further constructed trivalent designer cellulosome assembling two cellulases with an expansin-like protein, which further amplified the synergistic effect. The functional mechanism of the two expansin-like proteins were further explained by structural and sequence analysis. The special component of cellulosome may provide new materials for the industrial lignocellulose bioconversion.

\section{Materials and methods}

\section{Strains}

C. clariflavum DSM 19732, C. thermocellum ATCC 27405, Bacteroides cellulosolvens DSM 2933, Clostridium cellulolyticum H10 ATCC 35319, and B. subtilis 168 were either described in previous work (Cui et al. 2012; Hong et al. 2014) or obtained from the relevant culture collections. Genomic DNA was prepared from cells of the designated bacterial strains as described previously (Cui et al. 2012).

\section{Cloning of protein modules}

The module names, the corresponding original proteins, the residue ranges, and the source of the strains were summarized in Table 1. The DNA of each module except $C c$ EXL1298 and $C c$ EXL1862 was amplified by PCR from the corresponding genomic DNA using the corresponding primers (Supplementary Table S1). The DNAs of CcEXL1298 and CcEXL1862 were synthesized (Shanghai Sunny Biotechnology Co., Ltd., Shanghai, China) after the codon optimization of the corresponding genes (CCEXL1298: GenBank CP003065 region 1503156-1503839, CcEXL1862: GenBank CP003065 region 2143474-2144181) for expression in Escherichia coli. The PCR products of CcEXL1298, CcEXL1862, BsEXLX1 (GenBank AL009126 region: 2032930-2033550), cbm-cohT3 (GenBank CP000568 region: 3620482-3621657), docT (GenBank CP000568 region: 2482538-2482741), cohC1 (GenBank U40345 region: 3778-4221), docC (GenBank U30321 region: 2682-2864), and $\operatorname{doc} B$ (GenBank AF224509 region: 7337-7546) were cloned into the vector pET28aNS (Cui et al. 2013), while the PCR products of cel9K (GenBank CP000568 region: 516340-518739), ce9F (GenBank CP000568 region: 663872-665767), and cohB3 (GenBank AF224509 region: 8684-9127) were cloned into the vector pET30aNS (modified from pET30a (Merck Millipore, Billerica, MA, USA) by 
Table 1 Summary of module names, original proteins, residue ranges, and source of strains used in this study

\begin{tabular}{cccc}
\hline Module name & Original protein (Uniprot No.) & Residue range & Source of strain \\
\hline CcEXL1298 & Cloc1_1298 (G8LZP9) & $100-327(228 \mathrm{AA})$ & Clostridium clariflavum \\
CcEXL1862 & Clocl_1862 (G8LUU0) & $100-335(236 \mathrm{AA})$ & Clostridium clariflavum \\
BsEXLX1 & YoaJ (O34918) & $26-232(207 \mathrm{AA})$ & Bacillus subtilis \\
CBM-CohT3 & CipA (Q06851) & $323-714(392 \mathrm{AA})$ & Clostridium thermocellum \\
DocT & Cel48S (A3DH67) & $674-741(68 \mathrm{AA})$ & Clostridium thermocellum \\
CohC1 & CipC (Q45996) & $283-430(148 \mathrm{AA})$ & Clostridium cellulolyticum \\
DocC & CelCCF (P37698) & $662-722(61 \mathrm{AA})$ & Clostridium cellulolyticum \\
CohB3 & ScaB (Q6A564) & $345-492(148 \mathrm{AA})$ & Bacteroides cellulosolvens \\
DocB & ScaA (Q9FDJ9) & $2250-2319(70 \mathrm{AA})$ & Bacteroides cellulosolvens \\
Cel9K & Cel9K (A3DCH1) & $30-829(800 \mathrm{AA})$ & Clostridium thermocellum \\
Cel9F & Cel9F (P26224) & $29-660(632 \mathrm{AA})$ & Clostridium thermocellum \\
\hline
\end{tabular}

adding NheI and SpeI sites as well as five extra bases GCGGC between the NdeI and BamHI sites).

\section{Construction of the plasmids for expressing the chimeric proteins}

The chimeric proteins and the corresponding plasmids were listed in Table 2. The recombinant plasmids pET28aNSCcEXL1298, pET28aNS-CcEXL1862 and pET28aNSBsEXLX1 were digested by enzymes NheI and BamHI. Utilizing the isocaudamers NheI and SpeI, the fragments of $C c E X L 1298, C c E X L 1862$, and BsEXLX1 were ligated to the vector pET28aNS-docB linearized by SpeI and BamHI, resulting in the chimeric plasmid pET28aNS-doc $B$ $C c E X L 1298$, pET28aNS-docB-CcEXL1862, and pET28aNS-docB-BsEXLX1 for expressing chimeric proteins b-CcEXL1298 b-CcEXL1862 and b-EXLX1, respectively. The chimeric plasmids pET30aNS-cel9K-docC and
pET30aNS-celF-docT for expressing chimeric proteins Cel9K-c and Cel9F-t were constructed in a same way by inserting the fragments of $\operatorname{doc} C$ and $d o c T$ into the linearized vector pET30aNS-celK and pET30aNS-celF, respectively. Similarly, the fragment $c b m$-cohT3 was ligated to the linearized plasmid pET28aNS-cohC1, and then the fragment cohCl-cbm-cohT3 was ligated to the linearized plasmid pET30aNS-cohB3 resulting in the plasmid pET30aNScohB3-cohC1-cbm-cohT3 for expressing the chimeric scaffoldin cohB3-cohC1-cbm-cohT3 (Scaf BCT).

\section{Protein expression and purification}

Each plasmid was transformed into E. coli strain BL21 (DE3) cells (Transgen Biotech, Beijing, China) for protein expression. The proteins were purified by an affinity chromatography using a Histrap $^{\text {TM }}$ HP column (GE Healthcare, Uppsala, Sweden) followed by a gel filtration chromatography using a Superdex
Table 2 Summary of protein names, plasmids and their construction origins

\begin{tabular}{llll}
\hline Protein name & Chimeric plasmid & Fragment inserted & Linearized vector \\
\hline CcEXL1298 & pET28aNS-CcEXL1298 & CcEXL1298 & pET28aNS \\
CcEXL1862 & pET28aNS-CcEXL1862 & CcEXL1862 & pET28aNS \\
BsEXLX1 & pET28aNS-BsEXLX1 & BsEXLX1 & pET28aNS \\
CBM-CohT3 & pET28aNS-cbm-cohT3 & cbm-cohT3 & pET28aNS \\
DocT & pET28aNS-docT & docT & pET28aNS \\
CohC1 & pET28aNS-cohC1 & cohC1 & pET28aNS \\
DocC & pET28aNS-docC & docC & pET28aNS \\
CohB3 & pET30aNS-cohB3 & cohB3 & pET30aNS \\
DocB & pET28aNS-docB & docB & pET28aNS \\
b-CcEXL1298 & pET28aNS-docB-CcEXL1298 & CcEXL1298 & pET28aNS-docB \\
b-CcEXL1862 & pET28aNS-docB-CcEXL1862 & CcEXL1862 & pET28aNS-docB \\
b-BsEXLX1 & pET28aNS-docB-BsEXLX1 & BsEXLX1 & pET28aNS-docB \\
Ce19K-c & pET30aNS-cel9K-docC & docC & pET30aNS-cel9K \\
Cel9F-t & pET30aNS-cel9F-docT & docT & pET30aNS-cel9F \\
Scaf CT & pET28aNS-cohC1-cbm-cohT3 & cbm-cohT3 & pET28aNS-cohC1 \\
Scaf BCT & pET30aNS-cohB3-cohC1-cbm-cohT3 & cohC1-cbm-cohT3 & pET30aNS-cohB3 \\
\hline
\end{tabular}


75 column (GE Healthcare, Uppsala, Sweden) (Chen et al. 2014), except that Scaf BCT was purified by cellulose affinity chromatography as described previously (Yaron et al. 1995). Fractions containing purified proteins identified by SDS-PAGE analysis were pooled and concentrated. The concentration of each purified protein was estimated by the absorbance at $280 \mathrm{~nm}$ based on the molar extinction coefficient predicted from the amino acid sequence using the ProtParam tool (http://www. expasy.org/tools/protparam.html).

\section{Preparation of native cellulosomes}

The native cellulosomes were prepared from 2- and 4-days growth of $C$. thermocellum ATCC 27405 and C. clariflavum DSM 19732, respectively, by the affinity purification method (Morgenstern et al. 1992).

\section{Assembly of designer cellulosomes}

Molar equivalents of the desired dockerin-bearing proteins and Scaf BCT were mixed in a storage buffer $(50 \mathrm{mM}$ Tris$\mathrm{Cl}, 100 \mathrm{mM} \mathrm{KCl}, 10 \mathrm{mM} \mathrm{CaCl}_{2}, \mathrm{pH}$ 7.4), and incubated at room temperature for $0.5 \mathrm{~h}$ to ensure the complex formation. The proper assembly of the designer cellulosome was checked by non-denaturing PAGE and parallel SDS-PAGE (both have $12 \%$ separating phase gel) using the same sample.

\section{Enzyme activity measurement}

Enzyme activity was assayed by a previously reported procedure with modifications (Vazana et al. 2010). The enzymes were diluted into $200 \mu \mathrm{L}$ final volume with $100 \mathrm{mM}$ acetate buffer $(\mathrm{pH} 5.0)$ containing $2 \%(w / v)$ Avicel (Sigma-Aldrich, Shanghai, China), $24 \mathrm{mM} \mathrm{CaCl}_{2}$, and $4 \mathrm{mM}$ EDTA. The reaction tubes were incubated at $55{ }^{\circ} \mathrm{C}$ for $24 \mathrm{~h}$ under shaking at $170 \mathrm{rpm}$, and reactions were terminated by immersing the tubes in ice water. The samples were then centrifuged for $10 \mathrm{~min}$ at 14,000 rpm and $80 \mu \mathrm{L}$ of the supernatant was mixed with $160 \mu \mathrm{L}$ dinitrosalicylic acid reagent (DNS) (Miller 1959). The mixtures were boiled for $5 \mathrm{~min}$ and then the optical density at $540 \mathrm{~nm}$ was measured. The enzyme activity was calculated according to a standard curve prepared using glucose and presented as the concentration of glucose equivalents (i.e., reducing sugars, $\mu \mathrm{M}$ ). The concentration of native cellulosomes used in the experiments was $10 \mu \mathrm{g} / \mathrm{mL}$, while the concentrations of the expansin-like proteins, the designer cellulosomes, and their components were $0.2 \mu \mathrm{M}$. All assays were performed in triplicate and statistical significances were determined by $t$ test for the different treatments $(p<0.05)$.

\section{Homology modeling and structural analysis}

MODELLER version 9.10 (Sali and Blundell 1993) was used to construct the structural models of CcEXL1298 and CcEXL1862 by homology modeling. The crystal structures of $B s E X L X 1$ (PDB codes 3D30 and 4FER) were used as the templates. Amino acid sequence alignments were performed using ClustalX2 software (Larkin et al. 2007). Alignment figures were prepared using the Espript server (Robert and Gouet 2014). Structures were analyzed and visualized by the MolMol software (Koradi et al. 1996).

\section{Results}

Synergistic effect of expansin-like domains of Clocl_1298 and Clocl_1862 with the native cellulosomes on cellulose hydrolysis

According to sequence analysis, each of the two proteins Clocl_1298 and Clocl_1862 in C. clariflavum DSM 19732 possesses an $\mathrm{N}$-terminal signal peptide, a dockerin module with moderate sequence identity (39 and $36 \%$, respectively) to C. thermocellum Doc48S, and an expansin-like domain with a high degree of sequence identity ( 60 and $62 \%$, respectively) to $B s$ EXLX1 (Fig. 1a). To evaluate the molecular function of the expansin-like domains (denoted as $C c$ EXL1298 and $C c$ EXL1862) of the two proteins, the synergistic effect of $C c$ EXL1298 or CcEXL1862 with the native cellulosomes of $C$. clariflavum or $C$. thermocellum on cellulose hydrolysis was investigated. According to the mechanism proposed for the synergistic effect of expansin (see Introduction), low cellulase (cellulosomes here and designer cellulosomes in next section) concentration and short reaction time was used in the assays to observe its accelerating effect on cellulase activity, which will generally result in a low conversion yield $(\sim 1 \%)$ as indicated before (Georgelis et al. 2015). Similar to the results of previous studies about expansins or expansin-like proteins, no cellulose hydrolytic activity could be detected for CcEXL1298, CcEXL1862, and BsEXLX1 (Fig. 2a). However, each of them can significantly promote the cellulase activity of the native cellulosomes of $C$. thermocellum ATCC 27405 or C. clariflavum DSM 19732 using Avicel as substrate. The reducing sugar yield was about $15-42 \%$ greater than that of the control without expansin-like protein. Furthermore, CcEXL1298 showed 15-20\% higher synergistic effect than $C c$ EXL1862 while the synergistic effect of $B s$ EXLX1 was between them for both kinds of cellulosomes (Fig. 2b). These results indicated that the expansin-like domains of Clocl_1298 and Clocl_1862 possess the function of bacterial expansin when cellulosomes were used as the cellulases. 
Fig. 1 Schematic representation of proteins in this study. a The domain organization of the expansin-like proteins Clocl 1298 and Clocl_1862 in $C$. clariflavum. The residue numbers of each domain were labeled below. b Recombinant proteins used in this study. The cohesin and dockerin modules are colored in green, blue, and yellow according to their original species Ba. cellulosolvens, $C$. cellulolyticum, and $C$. thermocellum, respectively a

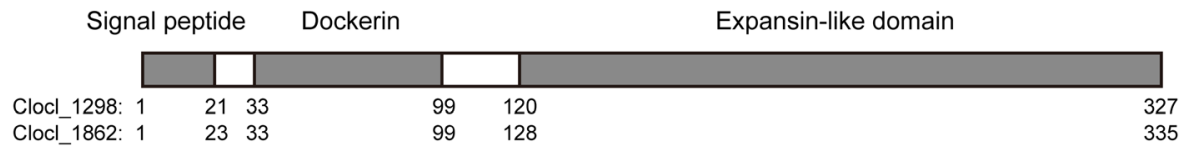

b

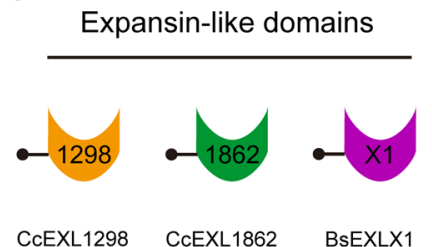

Chimeric enzymes

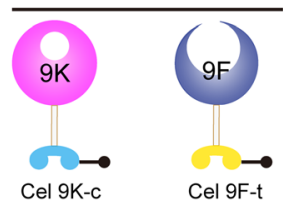

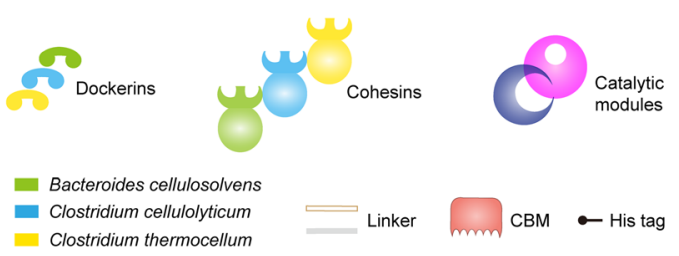

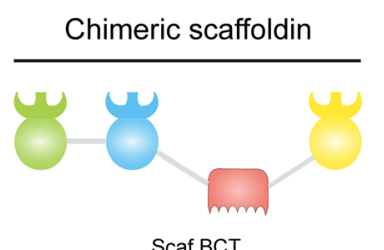

\section{Synergistic effect of CcEXL1298 and CcEXL1862 in designer cellulosome on the hydrolysis of cellulose}

The N-terminal dockerins of full-length proteins Clocl_1298 and Clocl_1862 indicated that they are also components of the cellulosome. In order to investigate whether the assembly of these expansin-like proteins and cellulosome could enhance their synergistic effect, we adopted the method of designer cellulosome to assemble the expansin-like protein and cellulases. A trivalent chimeric scaffoldin (Scaf BCT) was constructed using three cohesins from $\mathrm{Ba}$. cellulosolvens DSM 2933, C. cellulolyticum H10, and C. thermocellum ATCC
Fig. 2 Synergistic effect of CcEXL1298, CcEXL1862, and $B s$ EXLX1 with native cellulosomes on cellulose hydrolysis. a The cellulase activity of the non-catalytic proteins used in this study. $\mathbf{b}$ The cellulase activity of native cellulosomes of C. clariflavum DSM 1972 and C. thermocellum ATCC 27405 without (unfilled) or with the addition of expansin-like domains (orange, green, and purple for CcEXL1298, $C c$ EXL1862, and BsEXLX1, respectively). The assays were carried out with $2 \%(w / v)$ Avicel, $10 \mu \mathrm{g} / \mathrm{mL}$ cellulosomes, and $0.2 \mu \mathrm{M}$ expansin-like proteins at $55^{\circ} \mathrm{C}$ for $24 \mathrm{~h}$. Enzyme activity is defined as the concentration of released reducing sugars. Triplicates of each reaction were carried out, and standard deviations were indicated. Asterisk denotes $p<0.05$ (two-tailed $t$ test)
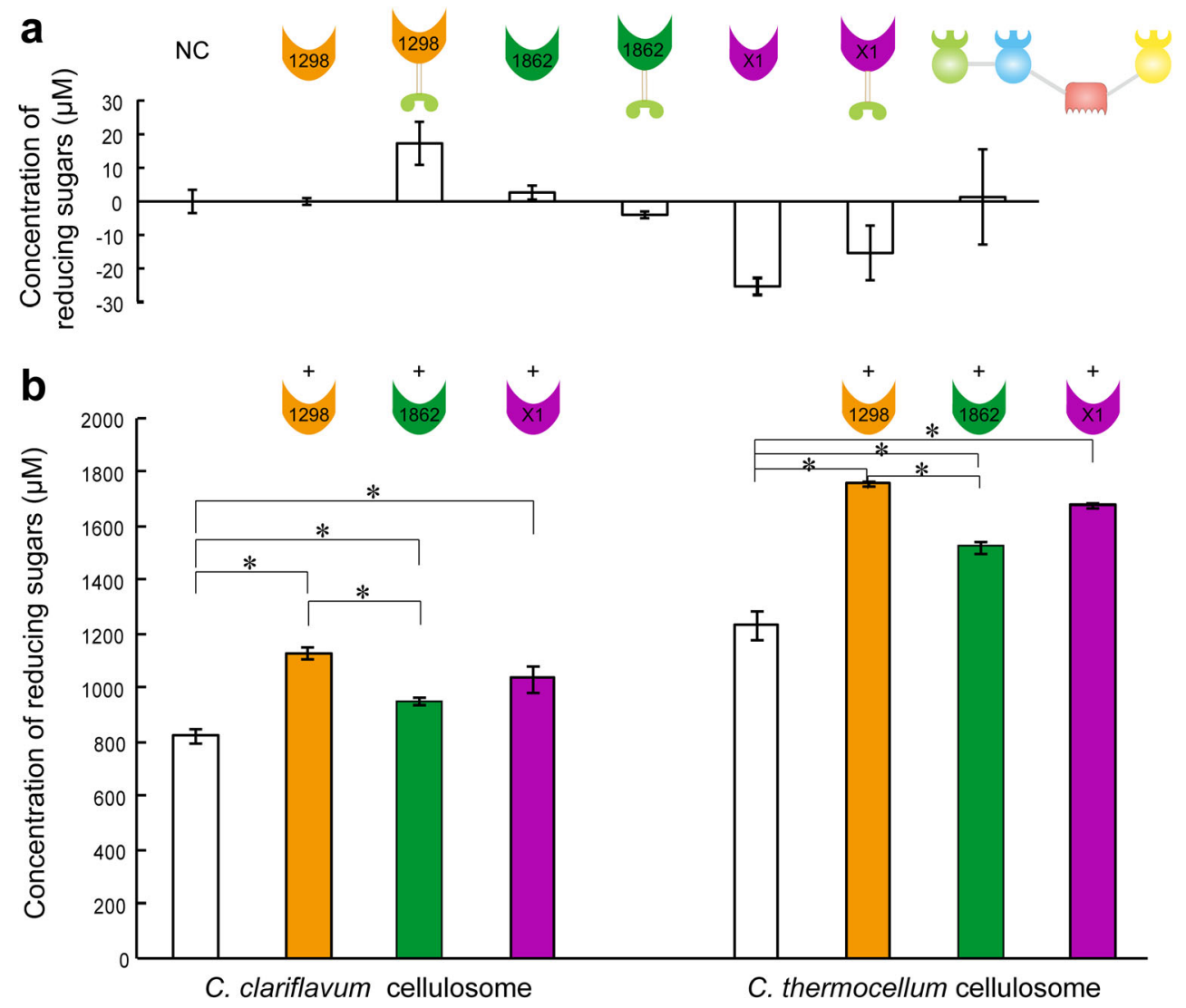
27405 , respectively, as well as the family 3 carbohydrate binding module (CBM3) from the scaffoldin CipA of $C$. thermocellum ATCC 27405. The glycoside hydrolase modules of two cellulases (Ce19K and Cel9F) from $C$. thermocellum ATCC 27405 were fused with the dockerin modules from C. cellulolyticum $\mathrm{H} 10$ and $C$. thermocellum ATCC 27405, respectively, forming the chimeric cellulases Cel9K-c and Cel9F-t. The expansin-like domains were fused with the dockerin module from Ba. cellulosolvens DSM 2933 forming the chimeric expansin-like proteins b-CcEXL1298, b-CcEXL1862, and b-BsEXLX1 (Fig. 1b). The dockerin modules are located at the N-termini of b-CcEXL1298, b$C c$ EXL1862 and b-BsEXLX1 and the C-termini of Cel9K-c and Cel9F-t according to the position of dockerins in the native proteins, and these dockerin modules are able to specifically bind to the corresponding cohesin modules on Scaf BCT. After mixing the purified recombinant chimeric proteins with a molar ratio of $1: 1$, the results of SDS-PAGE and nondenaturing PAGE indicated the correct assembly of the designer cellulosomes (Supplementary Fig. S1). As expected, the expansin-like domains lacking the dockerin modules cannot be integrated into the designer cellulosome, while the dockerin-bearing chimeric expansin-like proteins were integrated into the designer cellulosome.

We first verified the synergistic effect of the two cellulases in the designer cellulosome before involving expansin-like domains. Compared to the molar equivalents mixture of
Cel9K-c and Cel9F-t, the bifunctional designer cellulosome formed by Cel9K-c, Cel9F-t, and Scaf BCT in stoichiometric mixture showed significantly higher enzymatic activities on Avicel (Fig. 3a). The degree of synergy, defined as the amount of reducing sugar released from the designer cellulosome over the amount of reducing sugar released from free enzymes (Tsai et al. 2009), of the bifunctional designer cellulosome is 1.73 , which was similar to the results of previous reports (Fierobe et al. 2002).

Similar to the results of previous section, free expansin-like domains enhanced the activity of the bifunctional designer cellulosome with 15-20\% reducing sugar yield using Avicel $(2 \% w / v)$ as substrates (Fig. 3b). When the chimeric expansinlike proteins b-CcEXL1298, b-CcEXL1862, or b-BsEXLX1 was integrated into the tri-functional designer cellulosomes, the reducing sugar yield further increased by 5,11 , and $2 \%$, respectively (Fig. 3b). This result demonstrated that the integration of the expansin into the cellulosome can enhance the synergistic effect between the expansin-like protein and cellulases in lignocellulose degradation, which may explain why Clocl_1298 and Clocl_1862 bear a dockerin module in front of their expansin-like domain. Furthermore, CcEXL1862 showed the lowest synergistic effect among the three expansin-like domains when it is free in solution, which was consistent with the results in the previous section when the native cellulosome was tested. However, after the integration into the designer cellulosomes, the synergistic effects of three a

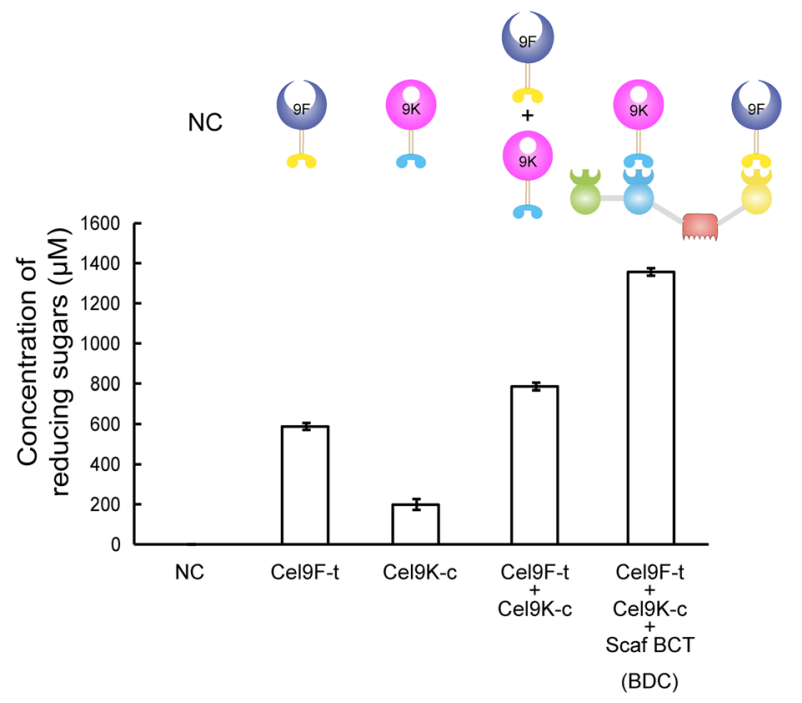

Fig. 3 Synergistic effect of $C c$ EXL1298, CcEXL1862 and BsEXLX1 in the designer cellulosomes on cellulose hydrolysis. a The synergistic effect of the bifunctional designer cellulosome assembled by Scaf BCT, Cel9Kc, and Cel9F-t. $N C$ negative control, $B D C$ bifunctional designer cellulosome. b The synergistic effect of $C c$ EXL1298, CcEXL1862, and $B s$ EXLX1 (in orange, green, and purple, respectively) with the b

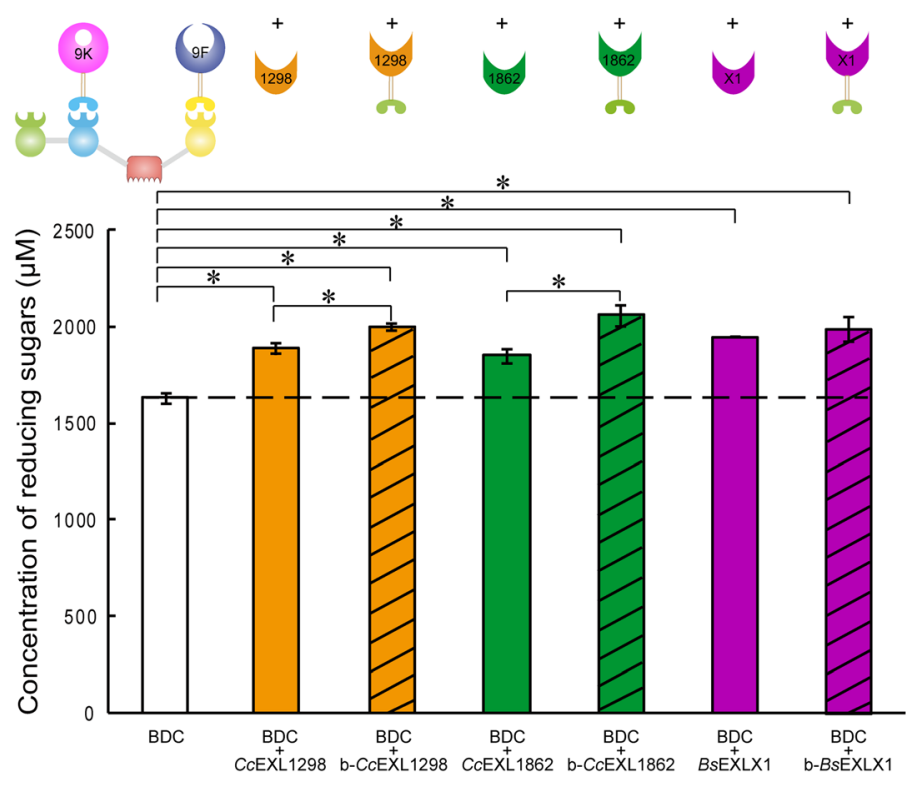

bifunctional designer cellulosome $(B D C)$ in mixture or assembly. The assays were carried out with $2 \%(w / v)$ Avicel, $0.2 \mu \mathrm{M}$ expansin-like proteins and designer cellulosome components at $55{ }^{\circ} \mathrm{C}$ for $24 \mathrm{~h}$. Enzyme activity is defined as the concentration of released reducing sugars. Triplicates of each reaction were carried out, and standard deviations were indicated. Asterisk denotes $p<0.05$ (two-tailed $\mathrm{t}$ test) 
expansin-like domains became less different, and bCcEXL1862 showed slightly higher synergistic effect than the other two expansin-like proteins.

Because some previous studies argued that the synergistic effects of bacterial expansin-like proteins are non-specific (Georgelis et al. 2015), we also examined the effect of several other proteins on the bifunctional cellulosome activity (Supplementary Fig. S2). A slight increase $(<10 \%)$ was observed for all of these proteins in the assays, reflecting the nonspecific effects of these proteins. Since the activity of the designer cellulosome was enhanced more than $20 \%$ after the integration of bacterial expansin-like proteins (Fig. 3b), the synergistic effects of bacterial expansin-like proteins in this study should be specific for these proteins.

\section{Sequence and structure analysis of Clocl_1298 and Clocl 1862}

We further performed sequence and structure analyses to investigate the functional mechanism of the two expansin-like proteins Clocl_1298 and Clocl_1862. First, the dockerin modules in the two proteins showed high sequence identity to the dockerins of other cellulosomal components (Fig. 4a). The putative calcium-binding sites and cohesin-binding residues are all conserved in the two dockerins, indicating they are functional cellulosomal components same as other cellulase components. We then analyzed the sequences of the expansinlike domains (Fig. 4b). They showed high sequence identity to the well-studied expansin-like protein $B s E X L X 1$, so we built structure models of $C c$ EXL1298 and $C c$ EXL1862 using homology modeling to analyze their structural details (Fig. 4c).

According to previous studies of BsEXLX1, the structure of the expansin-like protein contains two domains: D1 and D2 (Kerff et al. 2008). The domain D1 showed structural similarity to GH45 family glycoside hydrolases and several crucial residues (D71, Y73 and D82 in BsEXLX1) for the expansin activity have been identified (Kerff et al. 2008). These key residues are all completely conserved in CcEXL1298 and CcEXL1862, suggesting the domain D1 in CcEXL1298 and $C c$ EXL1862 has the function same as the domain D1 of BsEXLX1. The domain D2 of expansin-like protein showed structural similarity to type-A CBMs and included three conserved aromatic residues (W125, W126, Y157 in BsEXLX1) aligned in a plane for cellulose binding (Kerff et al. 2008). In CcEXL1298, the corresponding residues in the D2 domain become W245, W246, and C277, while in CcEXL1862 they are S251, W252, and W283, respectively. Therefore, only two of the three aromatic residues are conserved in CcEXL1298 and $C c$ EXL1862. In a previous study (Georgelis et al. 2011), the mutation of one to alanine in the three aromatic residues in the domain D2 of BsEXLX1 only partially weaken the activity. The C277 in CcEXL1298 and S251 in CcEXL1862 are residues easy to form hydrogen bonds, which may also partially restore the binding activity. Further, $C c$ EXL1862 possesses another aromatic residue F282 aligned in a plane with W252 and W283, which may also play roles in binding cellulose. In addition, the cellulose binding activity of Clocl 1298 and Clocl 1862 may be not as important as that of free expansins like BsEXLX1 because they are integrated in cellulosome which contains many cellulose binding modules and cellulases. Therefore, despite of the minor differences between these expansin-like domains, the two cellulosomal proteins Clocl_1298 and Clocl_1862 possess the most important structural and functional features of expansin and their synergistic effects are enhanced by the assembly of cellulosome.

\section{Discussion}

The composition of cellulosomes is dynamically regulated by the hosts to accommodate to the complicated structure and chemical components of lignocellulose (Raman et al. 2009; Blouzard et al. 2010; Kahel-Raifer et al. 2010). In addition to cellulases and hemicellulases, many other cellulosomal components have been found through the genomics analysis of cellulosome-producing bacteria (Zverlov et al. 2005; Izquierdo et al. 2012; Ben David et al. 2015) but their functions are not well defined. This study demonstrated the function of two cellulosomal expansin-like proteins, Clocl_1298 and Clocl_1862, from C. clariflavum. Both proteins were capable of promoting the hydrolytic activity of native cellulosomes of $C$. thermocellum or $C$. clariflavum, indicating their expansin-like functions. The synergistic effects were further enhanced when they were integrated into the cellulosomes. The sequence and structure analyses indicated that the cellulosomal expansin-like proteins bear functional dockerins and expansin-like domains with the mechanism same as the bacterial expansin-like protein BsEXLX1. Therefore, the cellulosomal expansin-like proteins may play roles in the lignocellulose degradation by $C$. clariflavum and they could be used to enhance the cellulosome activity in the lignocellulose bioconversion.

Bacterial expansins were found mainly in plant pathogens, soil inhabitants, or cellulose producers, suggesting their physiological roles in colonization or digestion of plant tissues (Georgelis et al. 2015). The finding of cellulosomal expansins provides more direct evidence to their physiological role in plant cell wall degradation. Recent cellulosome proteomics study revealed a low concentration of Clocl 1862 in $C$. clariflavum responding to various substrates, while no Clocl 1298 protein was identified (Artzi et al. 2015). This indicated these expansin-like proteins are not the key cellulosomal components in the cellulose degradation by $C$. clariflavum for nutrient intake. A new possibility is that the cellulosome plays certain roles in the progress of colonization during which expansin play more important roles. 


Dockerin
Clocl_1298
Clocl_1862
Clocl_0088

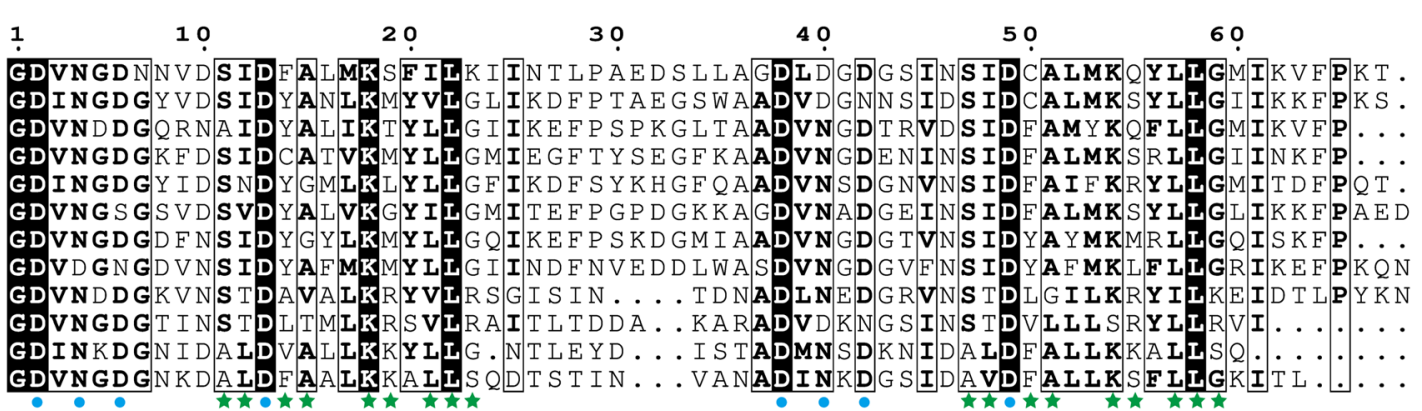

b
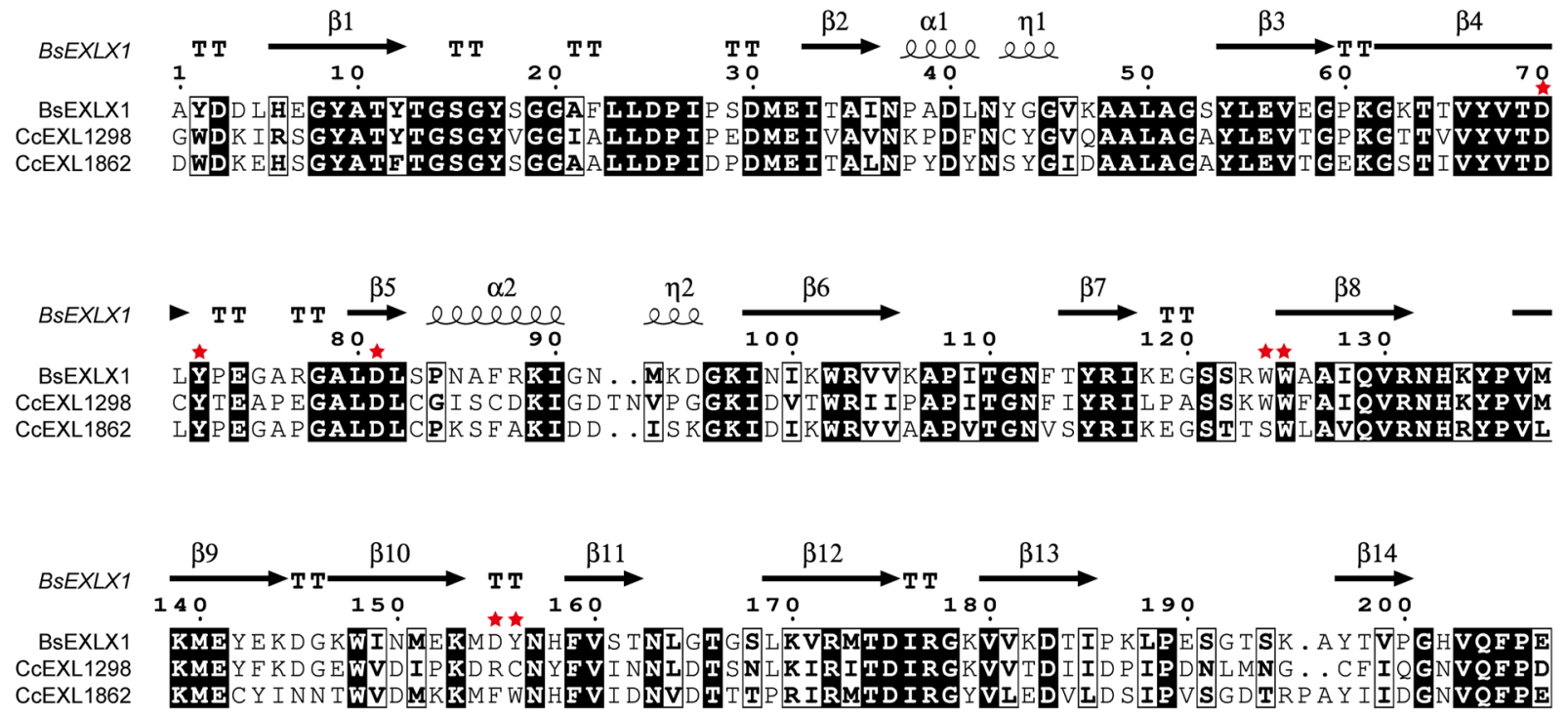

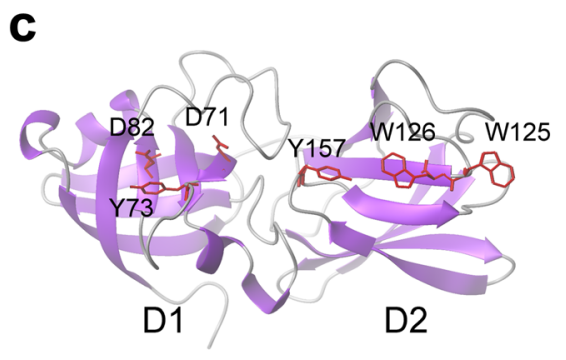

BsEXLX1

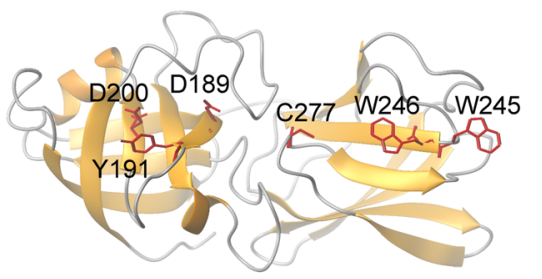

CCEXL1298

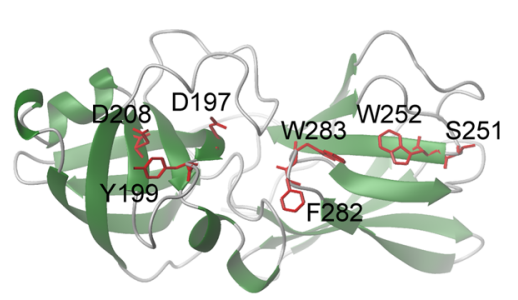

CcEXL1862
Fig. 4 Sequence and structure analysis of Clocl 1298 and Clocl 1862. a The sequence alignment of dockerin modules of $C$. clariflavum, $C$. thermocellum, and C. cellulolyticum. The putative calcium-binding and cohesin-binding residues were indicated by circles and stars, respectively, at the bottom of the alignment. b The sequence alignment of the expansin-like domains of BsEXLX1, Clocl_1298, and Clocl_1862. The

Though the cellulolytic functions of cellulosomes are well established, the existing of special components in cellulosomes suggests that cellulosomes may play additional functions. For example, homologs of $\mathrm{Cot} H$, a protein involved in spore coat assembly, have been found in cellulosomal genes of different species, such as C. clariflavum (Clocl 1061 and Clocl 4124), C. thermocellum (Cthe 0044 and Cthe_0239), Ruminococcus champanellensis (RUM_10710), Acetivibrio cellulolyticus (NCBI GI: 497937159, 497936844, and 497928905), and $\mathrm{Ba}$. cellulosolvens (NCBI GI: 739075399, 739075530, 739074220, key catalytic residues and substrate binding residues were indicated by red stars. $\mathbf{c}$ The crystal structure of BsEXLX1 (PDB code 3D30) and the structural models of $C c$ EXL1298 and $C c$ EXL1862. The crucial residues in $B s$ EXLX1 and corresponding residues in CcEXL1298 and $C c$ EXL1862 for the expansin activity in domain D1 and the substrate binding in domain D2 were shown in sticks

739073813, 739072938, 739072932, 739068809, 739064676). The multi-copy genes and wide existence of CotH suggest an importance functional correlation between cellulosomes and spore coat proteins. Similar to cellulosomal expansin-like proteins, the cellulosomal CotH proteins were also in a very low concentration or unidentified in proteomic studies (Gold and Martin 2007; Raman et al. 2009; Artzi et al. 2015; Ben David et al. 2015), suggesting that they are not key players in the lignocellulose degradation. Instead, the cellulosome may play certain role in CotH-related functions like spore assembly. How the 
cellulosomes play roles in the function of these non-cellulolytic proteins will be an interesting research topic in future.

Acknowledgments This study was supported by the National Basic Research Program of China (973 Program, Grant No. 2011CB707404) and the National Natural Science Foundation of China (grant nos. 31270784, 31300035 and 31470210).

\section{Compliance with ethical standards}

Ethical statement This article does not contain any studies with human participants or animals performed by any of the authors.

Conflict of interest The authors declare that they have no competing interests.

\section{References}

Artzi L, Dassa B, Borovok I, Shamshoum M, Lamed R, Bayer EA (2014) Cellulosomics of the cellulolytic thermophile Clostridium clariflavum. Biotechnol Biofuels 7:100. doi:10.1186/1754-6834-7-100

Artzi L, Morag E, Barak Y, Lamed R, Bayer EA (2015) Clostridium clariflavum: key cellulosome players are revealed by proteomic analysis. mBio 6(3):e00411-e00415. doi:10.1128/mBio.00411-15

Bayer EA, Belaich JP, Shoham Y, Lamed R (2004) The cellulosomes: multienzyme machines for degradation of plant cell wall polysaccharides. Annu Rev Microbiol 58:521-554. doi:10.1146/annurev. micro.57.030502.091022

Bayer EA, Lamed R, White BA, Flint HJ (2008) From cellulosomes to cellulosomics. Chem Rec 8(6):364-377. doi:10.1002/tcr.20160

Ben David Y, Dassa B, Borovok I, Lamed R, Koropatkin NM, Martens EC, White BA, Bernalier-Donadille A, Duncan SH, Flint HJ, Bayer EA, Moraïs S (2015) Ruminococcal cellulosome systems from rumen to human. Environ Microbiol 17(9):3407-3426. doi:10.1111/ 1462-2920.12868

Blouzard JC, Coutinho PM, Fierobe HP, Henrissat B, Lignon S, Tardif C, Pages S, de Philip P (2010) Modulation of cellulosome composition in Clostridium cellulolyticum: adaptation to the polysaccharide environment revealed by proteomic and carbohydrate-active enzyme analyses. Proteomics 10(3):541-554. doi:10.1002/pmic.200900311

Bunterngsook B, Mhuantong W, Champreda V, Thamchaipenet A, Eurwilaichitr L (2014) Identification of novel bacterial expansins and their synergistic actions on cellulose degradation. Bioresour Technol 159:64-71. doi:10.1016/j.biortech.2014.02.004

Bunterngsook B, Eurwilaichitr L, Thamchaipenet A, Champreda V (2015) Binding characteristics and synergistic effects of bacterial expansins on cellulosic and hemicellulosic substrates. Bioresour Technol 176:129-135. doi:10.1016/j.biortech.2014.11.042

Chen C, Cui Z, Xiao Y, Cui Q, Smith SP, Lamed R, Bayer EA, Feng Y (2014) Revisiting the NMR solution structure of the Cel48S type-I dockerin module from Clostridium thermocellum reveals a cohesinprimed conformation. J Struct Biol 188(2):188-193. doi:10.1016/j. jsb.2014.09.006

Cosgrove DJ (2000) Loosening of plant cell walls by expansins. Nature 407(6802):321-326. doi:10.1038/35030000

Cui GZ, Hong W, Zhang J, Li WL, Feng Y, Liu YJ, Cui Q (2012) Targeted gene engineering in Clostridium cellulolyticum $\mathrm{H} 10$ without methylation. J Microbiol Methods 89(3):201-208. doi:10.1016/ j.mimet.2012.02.015
Cui Z, Li Y, Xiao Y, Feng Y, Cui Q (2013) Resonance assignments of cohesin and dockerin domains from Clostridium acetobutylicum ATCC824. Biomol NMR Assign 7(1):73-76. doi:10.1007/s12104-012-9381-2

Cuiv PO, Gupta R, Goswami HP, Morrison M (2013) Extending the cellulosome paradigm: the modular Clostridium thermocellum cellulosomal serpin PinA is a broad-spectrum inhibitor of subtilisin-like proteases. Appl Environ Microbiol 79(19):61736175. doi:10.1128/aem.01912-13

Demain AL, Newcomb M, Wu JH (2005) Cellulase, clostridia, and ethanol. Microbiol Mol Biol Rev 69(1):124-154. doi:10.1128/mmbr 69.1.124-154.2005

Fierobe HP, Bayer EA, Tardif C, Czjzek M, Mechaly A, Belaich A, Lamed R, Shoham Y, Belaich JP (2002) Degradation of cellulose substrates by cellulosome chimeras. J Biol Chem 277(51):4962149630. doi:10.1074/jbc.M207672200

Georgelis N, Tabuchi A, Nikolaidis N, Cosgrove DJ (2011) Structurefunction analysis of the bacterial expansin EXLX1. J Biol Chem 286(19):16814-16823. doi:10.1074/jbc.M111.225037

Georgelis N, Nikolaidis N, Cosgrove DJ (2014) Biochemical analysis of expansin-like proteins from microbes. Carbohydr Polym 100:1723. doi:10.1016/j.carbpol.2013.04.094

Georgelis N, Nikolaidis N, Cosgrove DJ (2015) Bacterial expansins and related proteins from the world of microbes. Appl Microbiol Biotechnol 99(9):3807-3823. doi:10.1007/s00253-015-6534-0

Gold ND, Martin VJJ (2007) Global view of the Clostridium thermocellum cellulosome revealed by quantitative proteomic analysis. J Bacteriol 189(19):6787-6795. doi:10.1128/Jb.00882-07

Hong W, Zhang J, Feng Y, Mohr G, Lambowitz AM, Cui GZ, Liu YJ, Cui Q (2014) The contribution of cellulosomal scaffoldins to cellulose hydrolysis by Clostridium thermocellum analyzed by using thermotargetrons. Biotechnol Biofuels 7:80. doi:10.1186/1754-6834-7-80

Izquierdo JA, Goodwin L, Davenport KW, Teshima H, Bruce D, Detter C, Tapia R, Han S, Land M, Hauser L, Jeffries CD, Han J, Pitluck S, Nolan M, Chen A, Huntemann M, Mavromatis K, Mikhailova N, Liolios K, Woyke T, Lynd LR (2012) Complete genome sequence of Clostridium clariflavum DSM 19732. Stand Genom Sci 6(1):104 115. doi:10.4056/sigs. 2535732

Kahel-Raifer H, Jindou S, Bahari L, Nataf Y, Shoham Y, Bayer EA, Borovok I, Lamed R (2010) The unique set of putative membraneassociated anti-sigma factors in Clostridium thermocellum suggests a novel extracellular carbohydrate-sensing mechanism involved in gene regulation. FEMS Microbiol Lett 308(1):84-93. doi:10.1111/j. 1574-6968.2010.01997.x

Kerff F, Amoroso A, Herman R, Sauvage E, Petrella S, Filee P, Charlier P, Joris B, Tabuchi A, Nikolaidis N, Cosgrove DJ (2008) Crystal structure and activity of Bacillus subtilis YoaJ (EXLX1), a bacterial expansin that promotes root colonization. Proc Natl Acad Sci U S A 105(44):16876-16881. doi:10.1073/pnas.0809382105

Kim ES, Lee HJ, Bang WG, Choi IG, Kim KH (2008) A novel cellulase activity enhancing protein from Bacillus subtilis, a functional homo$\log$ of a plant expansin. J Biotechnol 136:S426-S426. doi:10.1016/j. jbiotec.2008.07.986

Kim ES, Lee HJ, Bang WG, Choi IG, Kim KH (2009) Functional characterization of a bacterial expansin from Bacillus subtilis for enhanced enzymatic hydrolysis of cellulose. Biotechnol Bioeng 102(5):1342-1353. doi:10.1002/bit.22193

Kim IJ, Ko HJ, Kim TW, Nam KH, Choi IG, Kim KH (2013) Binding characteristics of a bacterial expansin (BsEXLX1) for various types of pretreated lignocellulose. Appl Microbiol Biotechnol 97(12): 5381-5388. doi:10.1007/s00253-012-4412-6

Koradi R, Billeter M, Wuthrich K (1996) MOLMOL: a program for display and analysis of macromolecular structures. J Mol Graph 14(1):51-55. doi:10.1016/0263-7855(96)00009-4

Kumar R, Singh S, Singh OV (2008) Bioconversion of lignocellulosic biomass: biochemical and molecular perspectives. J Ind Microbiol Biotechnol 35(5):377-391. doi:10.1007/s10295-008-0327-8 
Larkin MA, Blackshields G, Brown NP, Chenna R, McGettigan PA, McWilliam H, Valentin F, Wallace IM, Wilm A, Lopez R, Thompson JD, Gibson TJ, Higgins DG (2007) Clustal W and clustal X version 2.0. Bioinformatics 23(21):2947-2948. doi:10.1093/bioinformatics/btm404

Lee HJ, Kim IJ, Kim JF, Choi IG, Kim KH (2013) An expansin from the marine bacterium Hahella chejuensis acts synergistically with xylanase and enhances xylan hydrolysis. Bioresour Technol 149: 516-519. doi:10.1016/j.biortech.2013.09.086

Meguro H, Morisaka H, Kuroda K, Miyake H, Tamaru Y, Ueda M (2011) Putative role of cellulosomal protease inhibitors in Clostridium cellulovorans based on gene expression and measurement of activities. J Bacteriol 193(19):5527-5530. doi:10.1128/jb.05022-11

Miller GL (1959) Use of dinitrosalicylic acid reagent for determination of reducing sugar. Anal Chem 31(3):426-428. doi:10.1021/ac60147a030

Morgenstern EM, Bayer EA, Lamed R (1992) Affinity digestion for the near-total recovery of purified cellulosome from Clostridium thermocellum. Enzym Microb Technol 14(4):289-292. doi:10. 1016/0141-0229(92)90153-F

Nikolaidis N, Doran N, Cosgrove DJ (2014) Plant expansins in bacteria and fungi: evolution by horizontal gene transfer and independent domain fusion. Mol Biol Evol 31(2):376-386. doi:10.1093/molbev/mst206

Rodriguez Jr M, Raman B, Pan C, Hurst GB, McKeown CK, Lankford PK, Samatova NF, Mielenz JR (2009) Impact of pretreated switchgrass and biomass carbohydrates on Clostridium thermocellum ATCC 27405 cellulosome composition: a quantitative proteomic analysis. PLoS One 4(4):e5271. doi:10.1371/journal.pone.0005271

Robert X, Gouet P (2014) Deciphering key features in protein structures with the new ENDscript server. Nucleic Acids Res 42 (Web Server issue):W320-W324. doi:10.1093/nar/gku316
Sali A, Blundell TL (1993) Comparative protein modelling by satisfaction of spatial restraints. J Mol Biol 234(3):779-815. doi:10.1006/ jmbi.1993.1626

Suzuki H, Vuong TV, Gong Y, Chan K, Ho CY, Master ER, Kondo A (2014) Sequence diversity and gene expression analyses of expansin-related proteins in the white-rot basidiomycete, Phanerochaete carnosa. Fungal Genet Biol 72:115-123. doi:10. 1016/j.fgb.2014.05.008

Tsai SL, Oh J, Singh S, Chen R, Chen W (2009) Functional assembly of minicellulosomes on the Saccharomyces cerevisiae cell surface for cellulose hydrolysis and ethanol production. Appl Environ Microbiol 75(19):6087-6093. doi:10.1128/aem.01538-09

Vazana Y, Morais S, Barak Y, Lamed R, Bayer EA (2010) Interplay between Clostridium thermocellum family 48 and family 9 cellulases in cellulosomal versus noncellulosomal states. Appl Environ Microbiol 76(10):3236-3243. doi:10.1128/aem.00009-10

Xu T, Li Y, He Z, Zhou J (2014) Dockerin-containing protease inhibitor protects key cellulosomal cellulases from proteolysis in Clostridium cellulolyticum. Mol Microbiol 91(4):694-705. doi:10.1111/mmi. 12488

Yaron S, Morag E, Bayer EA, Lamed R, Shoham Y (1995) Expression, purification and subunit-binding properties of cohesins 2 and 3 of the Clostridium thermocellum cellulosome. FEBS Lett 360(2):121124. doi:10.1016/0014-5793(95)00074-J

Zverlov VV, Kellermann J, Schwarz WH (2005) Functional subgenomics of Clostridium thermocellum cellulosomal genes: identification of the major catalytic components in the extracellular complex and detection of three new enzymes. Proteomics 5(14):3646-3653. doi:10.1002/pmic.200401199 